\title{
A simple method for latency analysis in signal detection tasks
}

\author{
DAVID NAVON \\ University of California, San Diego, La Jolla, California 92037
}

\begin{abstract}
It is proved that the difference between mean latency for incorrect responses and mean latency for correct ones in signal detection situations can be used as a measure for the separation between $\mathrm{SN}$ and $\mathrm{N}$ distributions, provided that certain plausible conditions are met. The applicability of this method to various paradigms is briefly discussed.
\end{abstract}

Investigators of signal detection often neglect to collect, analyze, or account for reaction time data. The same is true for experiments in various areas that may be analyzed within the framework of TSD terminology. ${ }^{1}$ The investigators who have analyzed latencies or have discussed latency analysis (e.g., Norman \& Wickelgren, 1969; Thomas \& Myers, 1972; Wickelgren, 1968; Yager \& Duncan, 1971) regarded latency as an inverse reflection of the subject's confidence. Thus, they obtained RT-ROC curves for "yes-no" tasks in basically the same way that ROC curves are derived from confidence rating (see Green \& Swets, 1966, pp. 40-43), only the RT scale was mapped into categories post hoc by means of choosing some arbitrary cutoff points. This technique requires that the experimenter record the latency to each response and then categorize the latencies, and that the final analysis will be done through plotting ROC curves. I will propose here a simpler technique that is straightforward to apply and will be shown to be easily derived from a few reasonable assumptions about the psychological variables underlying the experimental situation.

I will show, using a theorem proved by Thomas and Myers (1972), that under certain conditions the larger the separation between $\mathrm{N}$ (noise) and $\mathrm{SN}$ (signal plus noise) distributions, the larger is the difference between mean RT for false alarms $\left(\overline{\mathrm{RT}}_{\mathrm{FA}}\right)$ and mean RT for hits $\left(\overline{R T}_{H}\right)$, and so is the difference between mean $R T$ for misses $\left(\overline{\mathrm{RT}}_{\mathrm{M}}\right)$ and mean $\mathrm{RT}$ for correct rejections $\left(\overline{\mathrm{RT}}_{\mathrm{CR}}\right)$.

\section{ASSUMPTIONS}

(1) There is a continuous decision axis, $x$.

(2) Each situation is represented by a pair of density functions over $x$ that satisfy the condition $-d^{2} \log f(x) / d x^{2}>0$ (see Thomas, 1971). This property characterizes a large class of density functions, including,

This work was partially supported by Grant NS07454 from the National Institutes of Health. I am very much indebted to David Rumelhart for his enlightening suggestions and comments. The author is now at the Department of Psychology, University of Haifa, Haifa 31999 , Israel. for example, the normal, the gamma, and the exponential distributions. These functions will be denoted by $f_{N}(x)$ and $f_{S N}(x)$ for $N$ and $S N$, respectively.

(3) A unique criterion, c, corresponds to very combination of payoff matrix and set of prior probabilities.

(4) There exists a decreasing function, $L(x-c)$, defined over all positive real numbers that associates mean reaction time with deviations of sensory values, $x$, from the decision criterion, $c . L(x-c)$ may be regarded as the expectation of a distribution over all possible latencies associated with $\mathbf{x}-\mathrm{c}$, or as a unique latency associated with $\mathrm{x}-\mathrm{c}$, in which case a certain deviation of the sensory value from the criterion will always yield the same latency. It will be useful to associate with every criterion, $\mathrm{c}$, a latency function, $\mathrm{RT}_{\mathrm{c}}(\mathrm{x})$, defined over the decision axis. An example of a symmetrical $L$ function and two RT functions corresponding to it are plotted in Figure 1.

We will now prove that when we have different pairs of $(\mathrm{N}, \mathrm{SN})$ satisfying the condition that the $\mathrm{N}$ distributions may differ only in their expectations and the SN distributions may differ only in their expectations, then, given a fixed false-alarm rate or a fixed hit rate, the expression

$$
\frac{\int_{c}^{\infty} f_{N}(x) R T_{c}(x) d x}{\int_{c}^{\infty} f_{N}(x) d x}-\frac{\int_{c}^{\infty} f_{S N}(x) R T_{c}(x) d x}{\int_{c}^{\infty} f_{S N}(x) d x}
$$

is an increasing function of $d_{s}$, where $d_{s}$ in this case is defined as: $\left(\mu_{\mathrm{SN}}-\mu_{\mathrm{N}}\right) / \sigma_{\mathrm{SN}}$. That is, the larger the separation between the distributions, the langer the difference $\overline{\mathbf{R T}}_{\mathbf{F A}}-\overline{\mathrm{RT}}_{\mathbf{H}}$.

\section{PROOF}

Suppose we have two pairs of (N,SN) differing in $d_{3}$. Let us denote the distributions corresponding to the larger $d_{s}$ by the superscript $S$ (for "strong") and the distributions corresponding to the smaller $d_{s}$ by the superscript W (for "weak"). 

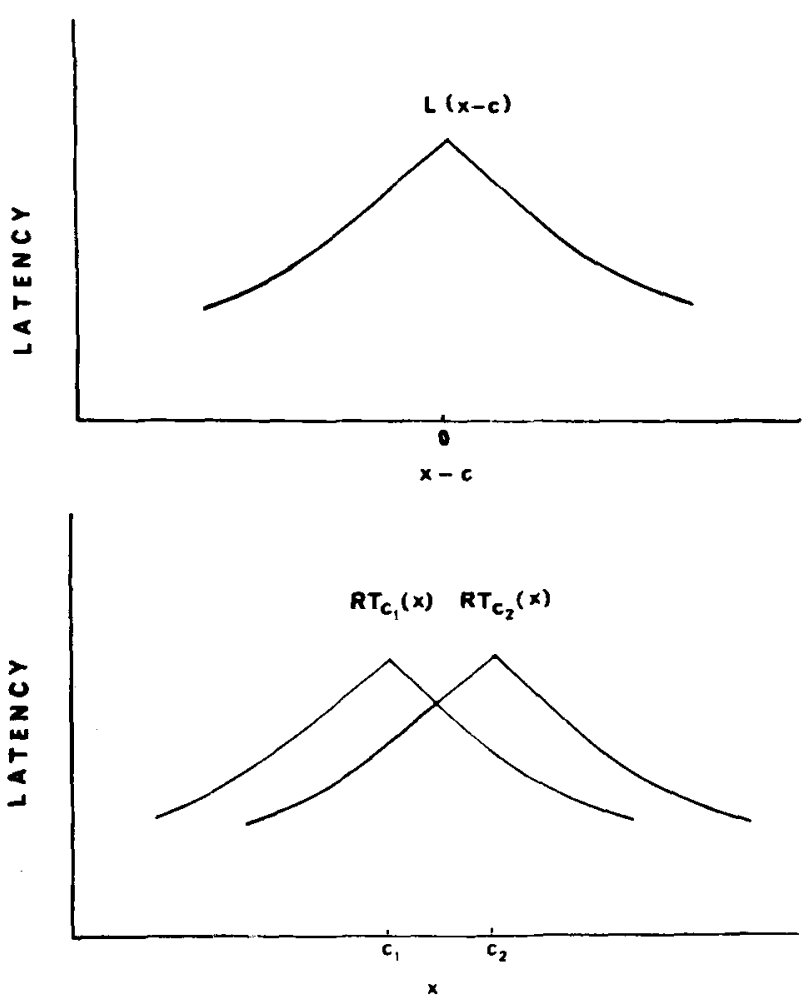

Figure 1. An example of two latency functions, $\mathrm{RT}_{\mathrm{c}_{1}}(\mathrm{x})$ and $\mathrm{RT}_{\mathrm{c}}(\mathrm{x})$, defined over the decision variable, $\mathrm{x}$, for different criteria $\left(c_{1}\right.$ and $\left.c_{2}\right)$. Both are derived from the same latency function, $L$, defined over the deviations of $x$ from the criterion.

Equal false-alarm rates can arise from either of two types of situations: (1) $\mu_{\mathrm{N}}^{\mathrm{S}}=\mu_{\mathrm{N}}^{\mathrm{W}}=\mu_{\mathrm{N}}$, and the criterion used in both cases is identical. (2) $\mu_{\mathrm{N}}^{\mathrm{S}} \neq \mu_{\mathrm{N}}^{\mathrm{W}}$ and $\mathrm{c}^{\mathrm{W}}=$ $\mathrm{c}^{\mathbf{S}}+\left(\mu_{\mathrm{N}}^{\mathrm{W}}-\mu_{\mathrm{N}}^{\mathrm{S}}\right)$.

We will restrict our proof to the first situation without any loss of generality. ${ }^{2}$

We thus want to show that

$$
\begin{aligned}
& \frac{\int_{c}^{\infty} f_{N}(x) R T_{c}(x) d x}{\int_{c}^{\infty} f_{N}(x) d x}-\frac{\int_{c}^{\infty} f_{S N}^{S}(x) R T_{c}(x) d x}{\int_{c}^{\infty} f_{S N}^{S}(x) d x} \\
& \quad>\frac{\int_{c}^{\infty} f_{N}(x) R T_{c}(x) d x}{\int_{c}^{\infty} f_{N}(x) d x}-\frac{\int_{c}^{\infty} f_{S N}^{W}(x) R T_{c}(x) d x}{\int_{c}^{\infty} f_{S N}^{W}(x) d x} .
\end{aligned}
$$

Since the first terms in both sides of the inequality are equal, this reduces to

$$
\frac{\int_{c}^{\infty} f_{S N}^{W}(x) R T_{c}(x) d x}{\int_{c}^{\infty} f_{S N}^{W}(x) d x}>\frac{\int_{c}^{\infty} f_{S N}^{S}(x) R T_{c}(x) d x}{\int_{c}^{\infty} f_{S N}^{S}(x) d x} .
$$

Since the variances of $f_{S N}^{S}(x)$ and $f_{S N}^{W}(x)$ are equal. we use the following notation:

$$
f(x)=f_{S N}^{W}(x)
$$

and

$$
\Delta=\left(\mu_{\mathrm{SN}}^{\mathrm{S}}-\mu_{\mathrm{N}}^{\mathrm{S}}\right)-\left(\mu_{\mathrm{SN}}^{\mathrm{W}}-\mu_{\mathrm{N}}^{\mathrm{W}}\right)
$$

so that

$$
f(x-\Delta)=f_{S N}^{S}(x) .
$$

Rewriting Inequality 2, we obtain

$$
\frac{\int_{c}^{\infty} f(x) R T_{c}(x) d x}{\int_{c}^{\infty} f(x) d x}>\frac{\int_{c}^{\infty} f(x-\Delta) R T_{c}(x) d x}{\int_{c}^{\infty} f(x-\Delta) d x} .
$$

The right side of the inequality equals

$$
\frac{\int_{c-\Delta}^{\infty} f(x) R T_{c-\Delta}(x) d x}{\int_{c-\Delta}^{\infty} f(x) d x},
$$

and recalling also the definitions of $\mathrm{RT}_{\mathrm{c}_{i}}$, we may rewrite Inequality 3 as

$$
\frac{\int_{c}^{\infty} f(x) L(x-c) d x}{\int_{c}^{\infty} f(x) d x}>\frac{\int_{c-\Delta}^{\infty} f(x) L(x-c+\Delta) d x}{\int_{c-\Delta}^{\infty} f(x) d x}
$$

Inequality 4 follows from a proof by Thomas and Myers (1972, Theorem 10). Thus we have completed our proof.

An analogous proof can be constructed for the case of equal hit rates. It can be shown also that the difference $\overline{\mathrm{RT}}_{\mathrm{M}}-\overline{\mathrm{RT}}_{C R}$ is positively related to $\mathrm{d}_{\mathbf{S}}$. Thus the difference between mean $\overline{\mathrm{RT}}$ for incorrect responses and mean $\overline{\mathrm{RT}}$ for correct responses is also positively related to $\mathrm{d}_{\mathrm{s}}$.

If the variance of the signal is positively correlated with its mean, namely stronger signals are associated with larger variance, the situation becomes less clear. Suppose we can show that $\overline{\mathrm{RT}}_{\mathrm{H}}$ for a given signal is not smaller than $\overline{\mathrm{RT}}_{\mathrm{H}}$ for a signal with the same mean but with larger variance. Then, since Inequality 2 is true for "strong" signals with the same variance as the "weak" ones, it must also hold for "strong" signals with larger variance. Let $\mathrm{fSv}(\mathrm{x})$ and $\mathrm{f} \mathrm{L}(\mathrm{x})$ denote, respectively, the densities of the signals with small and large variances. We want to find the conditions under which 


$$
\frac{\int_{c}^{\infty} f^{L V}(x) R^{2} T_{c}(x) d x}{\int_{c}^{\infty} f^{L V}(x) d x} \leqslant \frac{\int_{c}^{\infty} f^{S V}(x) R T_{c}(x) d x}{\int_{c}^{\infty} f^{S v}(x) d x}
$$

If the criterion lies at a point to the right of which $\mathrm{f}^{\mathrm{LV}}(\mathrm{x})-\mathrm{f}^{\mathrm{SV}}(\mathrm{x})$ is increasing in $\mathrm{x}$ (for the normal distribution, the smallest value satisfying this requirement is the mean), then Inequality 5 must be true, because in $\mathrm{fLV}^{\mathrm{L}}(\mathrm{x})$ large values of $\mathrm{RT}_{\mathrm{c}}(\mathrm{x})$ are weighted by smaller densities. However, if the criterion is lower, then the truth of Inequality 5 depends on the precise shape of $L$ and the location of $c$. The inequality does not hold only if the $\mathbf{L}$ function is extremely convex or the criterion is very low. For example, when the variance of $\mathrm{fsv}^{\mathrm{SV}}(\mathrm{x})$ is 1 and $\mathrm{L}(\mathrm{x}-\mathrm{c})$ is exponential with $\lambda=2$ (which decays very rapidly), then Inequality 5 still holds for every criterion that is not more than 1.5 standard deviations below the mean. Thus, it is almost always safe to assume that Inequality $I$ is true, even if the variances of the different signals cannot be believed to be identical.

\section{APPLICATIONS}

In what experimental paradigms may we expect the assumptions mentioned here to hold? Evidently none of these assumptions seems too strong in the context of pure detection tasks, where the noise distribution is usually assumed to be identical across signals, and the variances of the different SN distributions are often assumed to be identical. One has only to postulate the continuous theory of signal detection rather than a state model of the sort described, e.g., in Krantz (1969) or Luce (1963). When the signal detection theory is applied to such other paradigms as forced-choice recognition, "same-different" judgments, recall, etc., one should worry more about the validity of the assumptions. However, note that we do not have to assume $\sigma_{N}=\sigma_{S N}$, only that the variance is the same across signals and the same across noises; and, as shown earlier, this assumption is robust enough to withstand some severe violations. As for the assumption that either the false-alarm rates or the hit rates are identical in both experimental situations, it is up to the researcher to decide whether or not this assumption approximates well the real nature of the experimental situations.

The reported result suggests a very simple measure for comparing separation of distributions in different pairs $(\mathrm{N}, \mathrm{SN})$ satisfying the above assumptions, namely the difference between mean latency for incorrect responses and mean latency for correct ones. If one has the facilities for measuring reaction time, it is an almost costless way to buttress findings obtained from proportions data. Since parametric statistical tests are generally more powerful than nonparametric techniques, one may consider using latency data instead of proportions or $\mathrm{d}^{\prime}$ indices in order to save on the number of observations.
If the experimenter collects data under different payoff conditions and/or different prior probabilities conditions, he may want to present the data corresponding to each decision criterion separately rather than aggregating them across criteria. In this case, he may dispiay the data as an ROC curve relating $\overline{\mathrm{RT}}_{\mathrm{FA}}$ to $\overline{\mathrm{RT}}_{\mathrm{H}}$. It follows from Theorem 10 in Thomas and Myers that the higher the criterion for a given $\mathrm{d}_{\mathbf{S}}$, the greater are both $\overrightarrow{\mathrm{RT}}_{\mathrm{H}}$ and $\overrightarrow{\mathrm{RT}}_{\mathbf{F A}}$. Since we want to be able to compare different subjects or different sessions, it may be useful to plot the ROC curve in the unit square. This can be done by finding both the minimal $\overline{\mathrm{RT}}$ over the whole experiment $[\operatorname{Min}(\overline{\mathrm{RT}})]$ and the maximal $\overline{\mathrm{RT}}$ over the whole experiment $[\operatorname{Max}(\overline{\mathrm{RT}})]$, and then plotting for each decision criterion the point $(\mathrm{u}, \mathrm{v})$, where

$$
\begin{gathered}
u=\frac{\overline{\mathrm{RT}}_{\mathrm{H}}-\operatorname{Min}(\overline{\mathrm{RT}})}{\operatorname{Max}(\overline{\mathrm{RT}})-\operatorname{Min}(\overline{\mathrm{RT}})} \\
\mathrm{v}=\frac{\overline{\mathrm{RT}}_{\mathbf{F A}}-\operatorname{Min}(\overline{\mathrm{RT}})}{\operatorname{Max}(\overline{\mathrm{RT}})-\operatorname{Min}(\overline{\mathrm{RT}})} .
\end{gathered}
$$

The larger $d_{S}$ is, the farther should the curve be from the positive diagonal. A similar curve can be plotted for $\overline{\mathrm{RT}}_{\mathbf{M}}$ and $\overline{\mathrm{RT}}_{\mathbf{C R}}$. However, it is doubtful whether such plots reveal more information about the nature of the situation than an aggregated score. The reason is that latency is also a function of factors such as vigilance, practice, etc.; and since different criteria are used in different sessions, or at least in large blocks of trials, those factors may override any tendency correlated with the confidence of the subject in his responses.

\section{REFERENCES}

GreEN, D. M.. \& LuCE, D. R. Detection of auditory signals presented at random times. Perception \& Psychophysics, $1967,2,441-450$.

GreEN, D. M., \& Swets, J. A. Signal detection theory and psychophysics. New York: Wiley, 1966.

KraNtz, D. H. Threshold theories of signal detection. Psychological Review, 1969, 76, 308-324.

LUCE, R, D. A threshold theory for simple detection experiments. Psychological Review, 1963, 70, 61-79.

Norman, D. A., \& WICKElgRen, W. Strength theory of decision rules and latency in retrieval from short-term memory. Journal of Mathematical Psychology, 1969, 6, 192-208.

Tномаs, E. A. Sufficient conditions for monotone hazard rate-an application to latency-probability curves. Joumal of Mathematical Psychology. 1971, 8, 303-332.

Thomas, E. A., \& MYERs, J. L. Implications of latency data for threshold and nonthreshold models of signal detection. Journal of Mathematical Psychology, 1972, 9, 253-285.

WiCkElgren, W. Unidimensional strength theory and component analysis of noise in absolute and comparative judgements. Journal of Mathematical Psychology, 1968. 5. 102-122.

YAGER, D.. \& DUNCAN. I. A. Signal detection analysis of luminance generalization in goldtish using latency as a graded response measure. Perception \& Psychophysics. 1971. 9. $35.3-355$. 


\section{NOTES}

1. Excluding vigilance paradigms or random presentation experiments (e.g., Green \& Luce, 1967), where the nature of the paradigm dictates using latencies as the dependent variable.

2. Since the variance of the $\mathrm{N}$ distribution is the same in any case, a situation, $\beta$, of the second type is totally equivalent for the purpose of our proof to a situation, $\alpha$, of the first type where:

$$
\begin{aligned}
& \delta=\mu_{\mathrm{N}}^{\mathrm{W}, \beta}-\mu_{\mathrm{N}}^{\mathrm{S}, \beta} \\
& \mathrm{f}_{\mathbf{N}}^{\mathrm{S}, \alpha}(\mathrm{x})=\mathrm{f}_{\mathrm{N}}^{\mathbf{S}, \beta}(\mathrm{x}-\delta)
\end{aligned}
$$

$$
\begin{aligned}
& \mathrm{f}_{\mathrm{S}}^{\mathrm{S}, \alpha}(\mathrm{x})=\mathrm{f}_{\mathrm{SN}}^{\mathrm{S}, \beta}(\mathrm{x}-\delta) \\
& \mathrm{f}_{\mathrm{N}}^{\mathrm{W}, \alpha}(\mathrm{x})=\mathrm{f}_{\mathrm{N}}^{\mathrm{W}, \beta}(\mathrm{x}) \\
& \mathrm{f}_{\mathrm{SN}}^{\mathrm{W}, \alpha}(\mathrm{x})=\mathrm{f}_{\mathrm{SN}}^{\mathrm{W}, \beta}(\mathrm{x}) \\
& \mathrm{c}^{\alpha}=\mathrm{c}^{\mathrm{W}, \beta}=\mathrm{c}^{\mathrm{S}, \beta+\delta} .
\end{aligned}
$$

(Received for publication February 26, 1975; revision received April 18, 1975.) 